\title{
Bases anatómicas del sueño
}

\section{Anatomical basis of sleep}

\author{
J. L. Velayos, F. J. Moleres, A. M. Irujo, D. Yllanes, B. Paternain
}

\section{RESUMEN}

El sueño es un estado biológico activo, periódico, en el que se distinguen las etapas NREM y REM, que se alternan sucesivamente durante la noche. Intervienen los relojes biológicos en la modulación del sistema, así como neurotransmisores específicos. Se trata de una red neuronal compleja, en la que intervienen diversas zonas del sistema nervioso central. Los procesos oníricos están controlados además de forma neural.

Se resume la historia de las investigaciones sobre el tema, desde el siglo XIX hasta nuestra época. Hay que destacar los recientes descubrimientos de Lugaresi y su equipo, que, al describir el insomnio familiar grave, dieron importancia al núcleo dorsomedial del tálamo en la instauración de la fase de sueño profundo. Al grupo de Reinoso se debe el hallazgo de que el "director de orquesta" en la instauración del sueño REM es la zona ventral paramediana del núcleo reticular pontino oral

Palabras clave. Sueño. Vigilia. Centros neurales. REM. NREM.

An. Sist. Sanit. Navar. 2007; 30 (Supl. 1): 7-17.

\begin{abstract}
Sleep is an active and periodic biological state composed of NREM and REM phases, which alternate during the night. Both biological clocks and specific neurotransmitters are involved in the modulation of this system. It is a complex neuronal network in which several areas of the central nervous system are involved. The oneiric processes are also controlled neurally.

This work summarises the history of the investigations on this topic from the $19^{\text {th }}$ century to date. It is worth mentioning the recent findings of Lugaresi and colleages who described fatal familial insomnia, a disease that helped to show the importance of the mediodorsal thalamic nucleus in the genesis of slow-wave sleep. Reinoso's group found out that the paramedian ventral area of the oral pontine reticular nucleus is the conductor in the establishment of REM sleep.
\end{abstract}

Key words. Sleep. Wakefulness. Neural centres. REM. NREM.
Departamento de Anatomía. Facultad de Medicina. Universidad de Navarra. Pamplona

\author{
Correspondencia: \\ José Luis Velayos Jorge \\ Departamento de Anatomía \\ Facultad de Medicina \\ Universidad de Navarra \\ $\mathrm{C} /$ Irunlarrea, 1 \\ 31008 Pamplona \\ Tfno. 948425600 \\ Fax 948425649 \\ E-mail: jvelayos@unav.es
}




\section{EL SUEÑO: ASPECTOS GENERALES}

Todos los animales, incluidos los unicelulares, tienen períodos de actividad y de reposo, habiendo en este último una inmovilidad relativa. Por otra parte, según se avanza en la escala filogenética, el proceso del sueño es cada vez más complejo.

El sueño, biológicamente, no es una falta total de actividad, sino que se puede considerar como un estado biológico concreto, un estado conductual, según se viene diciendo desde los años ochenta del pasado siglo. En el sueño se precisa de un ambiente y una postura adecuados, que son variables en distintas especies: hay animales que pueden dormir de pie, y otros que pueden hacerlo con los ojos abiertos. En contraposición al coma, el estado de sueño es reversible en respuesta a estímulos adecuados y genera cambios electroencefalográficos que lo distinguen del estado de vigilia. La disminución en la motricidad de la musculatura esquelética y en el umbral de reactividad a estímulos son otras dos características de este estado.

El sueño es periódico y en general espontáneo, y se acompaña en el hombre de una pérdida de la conciencia vigil. Sin embargo, aun cuando el hombre tenga sueño, puede, voluntariamente, no dormir.

El sueño tiene distintos grados de profundidad, y se presentan modificaciones fisiológicas concretas en cada una de las etapas del mismo. Para el estudio de los cambios funcionales que se dan durante el sueño se atiende a unas variables que se denominan indicadores del sueño: el electroencefalograma (EEG), los movimientos oculares y el tono muscular (la polisomnografía es el registro de los tres indicadores) $)^{1-3}$.

\section{ETAPAS DEL SUEÑO: ASPECTOS ANATÓMICOS GLOBALES}

Según estos indicadores, se distinguen varias etapas en el sueño:

La etapa I, de somnolencia o adormecimiento, en que tiene lugar la desaparición del ritmo alfa del EEG (típico del estado de vigilia), hay tono muscular y no hay movimientos oculares o, si los hay, son muy lentos.

La etapa II - III, de sueño ligero, se caracteriza por una disminución aún mayor del ritmo electroencefalográfico, con la aparición de los típicos husos de sueño y los complejos $\mathrm{K}$, fenómenos de los que es responsable el núcleo reticular del tálamo; sigue existiendo tono muscular, y no hay movimientos oculares.

La etapa IV, de sueño profundo, presenta un ritmo electroencefalográfico menor, no hay movimientos oculares y el tono muscular se mantiene o puede estar muy disminuido. En la instauración de esta fase del sueño intervienen, entre otras estructuras, la corteza prefrontal y el núcleo dorsomedial del tálamo. El Insomnio Familiar Grave es una enfermedad de tipo priónico y evolución fatal que fue descrita por primera vez por Lugaresi y su equipo en los años ochenta ${ }^{4}$, cuyo estudio permitió descubrir la importancia de tal estructura talámica para la instauración del sueño lento o profundo. Es la fase del sueño más reparadora. Hay movimientos organizados del dorso; el individuo da vueltas en la cama, cambia de postura. Esta fase dura aproximadamente un $25 \%$ del total del tiempo del sueño.

Las etapas I a IV se denominan en su conjunto sueño no REM (NREM).

La siguiente etapa es la de sueño paradójico, que se caracteriza por una actividad EEG que recuerda al estado de vigilia (por eso se habla de sueño paradójico), debida a una activación cortical por parte de estructuras encefálicas profundas, como es la formación reticular activadora. Fue descubierto por Kleitman y Aserinsky, junto con Dement ${ }^{5}$, en los años cincuenta del siglo pasado. Hay una desincronización del EEG, que se asemeja a una situación de vigilia, de alerta. Se observan movimientos oculares rápidos (también se habla de sueño MOR, de movimientos oculares rápidos o sueño REM, de rapid eye movements), dependientes de la actividad de estructuras profundas tales como la formación reticular pontina. Se produce una atonía (desaparición del tono muscular), de lo que son responsables estructuras como la formación reticular bulbar, el 
locus coeruleus, etc. El músculo diafragma sigue manteniendo el tono, y contrayéndose, permitiendo la respiración. La fase de sueño REM constituye un $25 \%$ del sueño total. En el recién nacido, el sueño REM constituye el $50 \%$ del tiempo total de sueño. El tiempo de vigilia va aumentando con la edad, cada vez se duerme menos, y cada vez hay menos sueño REM. Sólo existe sueño REM en los mamíferos, excepto el conejo macho, el oso hormiguero y el delfín de nariz en botella. Parece ser, en líneas generales, que el sueño paradójico se produce, filogenéticamente, cuando la corteza cerebral está más desarrollada. De la instauración del sueño REM es responsable el tronco del encéfalo, concretamente un grupo de neuronas que también descargan en la vigilia (el centro nodal es el núcleo reticular pontino oral, cuyas porciones ventral y paramediana reciben conexiones de múltiples estructuras relacionadas con el control del ciclo vigilia-sueño), produciendo una activación de los sistemas colinérgicos.

Las fases de sueño NREM y REM se alternan sucesivamente, cuatro a cinco veces por la noche. En total, la fase de sueño NREM dura unas 6 horas; y la fase de sueño REM, dos horas, por término medio. Es más fácil despertar al sujeto en la fase de sueño REM que en la fase NREM. Los medicamentos antidepresivos reducen el sueño REM y las benzodiacepinas acortan o suprimen las fases III y IV.

El metabolismo cerebral y en consecuencia la temperatura cerebral disminuye con la profundidad del sueño NREM. Sin embargo, en el sueño REM pueden incrementarse estas cifras con respecto al estado de vigilia, ya que hay una activación de la corteza cerebral.

En el sueño NREM se da una progresiva desactivación de la formación reticular activadora junto a una inhibición de las neuronas relé talámicas. Todo esto quiere decir que las estimulaciones sensoriales han de tener un umbral determinado para provocar el despertar, ya que en el sueño, y sobre todo en la fase de sueño profundo, no hay una concienciación de lo sensorial. Sin embargo, es curioso que se pueda dar un despertar ante situaciones determina- das: la madre se despierta cuando su bebé necesita algo, aun cuando no le despierten otros estímulos más potentes; algunas personas pueden despertarse a una hora predeterminada, con gran precisión. Deben de jugar un papel en este sentido sistemas de inhibiciones que desconocemos aún.

\section{RELOJES BIOLÓGICOS}

El sueño es por lo tanto un estado dinámico, en el que se activan e inhiben distintas zonas del encéfalo, con las repercusiones funcionales que se han indicado. Obedece a un ritmo biológico, circadiano (cada 24 horas), relacionado con el ritmo día-noche, nictemeral, al que se ajusta el proceso. De por sí, el ritmo sueño-vigilia es cada 25-29 horas, según se ha estudiado experimentalmente en voluntarios encerrados en una habitación a la que no llegan las influencias exteriores. Pero la presión del sueño aumenta en torno a las dos de la tarde, lo que explica que sea fisiológico sentir sueño después de comer. La voluntad puede evitar el dormir después de comer.

Existen unos relojes biológicos en el sistema nervioso central. Uno de ellos, situado en el hipotálamo (núcleo supraquiasmático), establece el ritmo sobre los otros relojes biológicos, situados caudalmente, y hace que el sueño NREM y el sueño REM duren un tiempo fijado. Intervienen en su regulación no sólo, y de modo fundamental, los impulsos retinianos, sino también otras influencias, como es el $\mathrm{pH}$ de la sangre o la glucemia. El núcleo supraquiasmático no es responsable en sí mismo del ritmo vigilia - sueño, pero sí forma parte de las redes neurales implicadas en el proceso, redes neurales sobre las que deben de actuar diversos sistemas para hacer que el proceso quede anulado por un tiempo, y al fin y al cabo retrasado. Las conexiones eferentes del núcleo supraquiasmático son muy profusas, e incluso bilaterales: hacia el hipotálamo posterior, región preóptica, núcleos del septo, núcleo paratenial, núcleo paraventircular del tálamo, núcleo ventral lateral del tálamo y glándula pineal ${ }^{6-8}$.

El proceso del ciclo vigila-sueño está regulado por una red neuronal compleja en 
la que intervienen diversas zonas del sistema nervioso central, a base de activaciones y de inhibiciones, cuyo resultado es la vigila o el sueño. Dentro del sueño, la fase de sueño REM es regulada por una complicada red neural en la que intervienen diversos neurotransmisores. Una explicación simplificada no es posible (Tabla 1, Fig. 1).
Se trata de un sistema homeostático en el que el sueño llama a la vigilia y la vigila al sueño, sistema que voluntariamente puede alterarse: un joven californiano de 23 años estuvo 288 horas sin dormir. La privación del sueño lleva a la muerte, sobre todo debido a la falta de la fase profunda del sueño. La privación del sueño

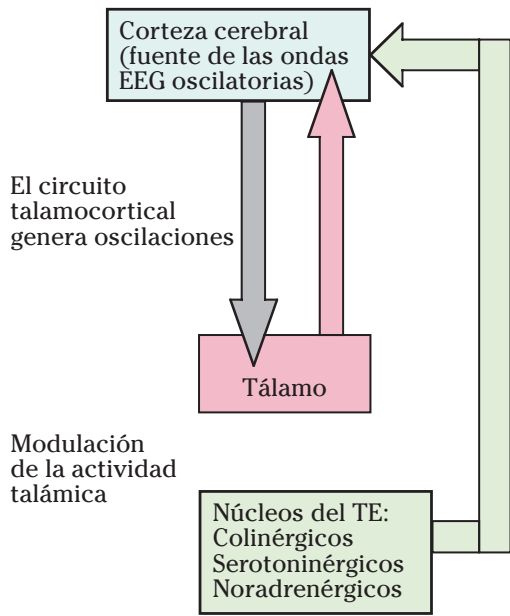

El control del sueño y la vigilia depende de la modulación del tálamo y de la corteza por el tronco del encéfalo (TE).

Los principales componentes del sistema modulador son los núcleos colinérgicos,

Modulación de la actividad cortical setoninérgicos.

Estas estructuras tienen proyecciones tanto a la corteza como al tálamo, donde tienen efectos directos e indirectos sobre la función cortical.

Figura 1. Circuitos implicados en la regulación del ritmo vigilia-sueño

Tabla 1. Resumen de los mecanismos celulares implicados en el sueño y la vigilia.

\begin{tabular}{|c|c|c|}
\hline $\begin{array}{l}\text { Núcleos del tronco del encéfalo } \\
\text { responsables }\end{array}$ & Neurotransmisor involucrado & $\begin{array}{l}\text { Estado de actividad de las neuronas } \\
\text { correspondientes del tronco } \\
\text { del encéfalo }\end{array}$ \\
\hline \multicolumn{3}{|l|}{ Vigilia } \\
\hline $\begin{array}{l}\text { Núcleos colinérgicos de la unión } \\
\text { ponto-mesencefálica }\end{array}$ & Acetilcolina & Activas \\
\hline Locus coeruleus & Noradrenalina & Activas \\
\hline Núcleos del rafe & Serotonina & Activas \\
\hline $\begin{array}{l}\text { Sueño no-REM } \\
\text { Núcleos colinérgicos de la unión } \\
\text { ponto-mesencefálica }\end{array}$ & Acetilcolina & Inactivas \\
\hline Locus coeruleus & Noradrenalina & Inactivas \\
\hline Núcleos del rafe & Serotonina & Inactivas \\
\hline \multicolumn{3}{|l|}{ Sueño REM CONECTADO } \\
\hline $\begin{array}{l}\text { Núcleos colinérgicos de la unión } \\
\text { ponto-encefálica }\end{array}$ & Acetilcolina & $\begin{array}{l}\text { Activas (ondas } \\
\text { pontogeniculooccipitales) }\end{array}$ \\
\hline Núcleos del rafe & Serotonina & Inactivas \\
\hline \multicolumn{3}{|l|}{ Sueño REM desconectado } \\
\hline
\end{tabular}


REM da lugar a alteraciones hipotalámicas, con agresividad, aumento del peso y finalmente muerte, según se ha visto experimentalmente.

\section{LOS ENSUEÑOS: BASES ANATÓMICAS}

Durante el sueño hay actividad mental: se trata de las experiencias oníricas, los ensueños, que pueden relatarse en una proporción del $80 \%$ al despertar al sujeto después de pasada una fase de sueño paradójico. Por lo tanto, el sueño no significa falta de vida ni falta de actividad mental. Los sueños de la fase REM son de tipo cinematográfico y en color. Algunos piensan que el movimiento de los ojos en esta fase REM va en seguimiento de las imágenes visuales del ensueño. También se sueña durante la fase de sueño profundo, pero estos sueños son más bien de tipo abstracto. El procesamiento de las imágenes de los ensueños, según los hallazgos clínicos, se hace de forma preferente en la corteza occipitotemporal.

Para Hobson ${ }^{9-11}$, en el sueño REM habría una activación del sistema reticular activador del tronco del encéfalo y del prosencéfalo basal, estructuras que también están activas en la vigilia. Para este autor, estructuras como los núcleos talámicos relé, que son los que reciben la estimulación sensorial, quedarían activados, lo que contribuiría a la aparición de las diversas modalidades psicológicas que aparecen en los sueños. Las estructuras límbicas, tales como la amígdala y la corteza cingular, también estarían activadas, lo que explicaría los fenómenos emotivos durante la fase de sueño REM. Los ganglios basales y el cerebelo, que regulan la motricidad, también activados, explicarían los movimientos ficticios de los sueños en la fase REM. Están activas cortezas asociativas tales como las del lobulillo parietal inferior y de la corteza occipitotemporal, todo lo cual explica las imágenes visuales de esta fase del sueño. La corteza prefrontal, importante en los procesos mentales, está inhibida, lo cual puede explicar la falta de lógica en los razonamientos que se experimentan en el sueño. Precisamente, como dice Reino$\mathbf{s o}^{12}$, las mismas estructuras que son nece- sarias para la consolidación de la memoria, tales como el tálamo medial, la amígdala, el hipocampo, las estructuras parahipocampales, la corteza orbitofrontal y cortezas asociativas monomodales están activas en el sueño REM.

En el sueño NREM, en cambio, hay una inhibición de las zonas que están activadas en el sueño REM y activación de las inhibidas, lo que puede explicar que en tal sueño NREM haya ensoñaciones más de tipo abstracto que en el sueño REM. El despertar en esta fase da lugar a estados confusionales y un rápido retorno al sueño.

\section{OTROS FENÓMENOS}

Ocurren otros fenómenos durante el proceso del sueño: por ejemplo, secreción de hormonas sexuales y de la hormona de crecimiento, sobre todo en el sueño NREM, de ahí la importancia del sueño en los niños y adolescentes. La fase de sueño REM es importante además para los procesos de memoria; parece ser que la consolidación de la memoria y la eliminación de los datos "inútiles" se hace durante la fase REM. Precisamente en los ancianos la duración del sueño REM disminuye bastante, en cambio en el feto, la fase REM ocupa la mayor parte del tiempo de sueño. Conforme avanza la edad, el estado de vigilia va aumentando en duración; cada vez se duerme menos, y cada vez hay menos sueño REM. Independientemente del sueño, la temperatura corporal cae en la madrugada. En el sueño REM hay tumefacción del pene, con independencia del tipo de ensueños.

Se puede resumir que el sueño es un estado fisiológico activo, en que participan diversas estructuras encefálicas, formando una red neuronal, en que se dan activaciones e inhibiciones complejas, con una regulación cíclica, y sobre la que puede actuar la voluntad, modulando y estableciendo determinadas actitudes, comportamientos. Por ejemplo, cada persona tiene su "liturgia", sus hábitos para dormir: unos necesitan leer un poco antes de conciliar el sueño; otros han de tomar un vaso de agua; otros, precisan de una oscuridad total; otros, de cierto grado de luminosidad, etc. Los sueños son distintos de unas 
personas a otras; así por ejemplo, las personas creativas tienen sueños particularmente ricos, mientras que las más simples, los tienen más elementales. Por tanto, la impronta personal también deja su huella en estos procesos.

\section{HACIENDO HISTORIA (ANATOMÍA DEL SUEÑO)}

Podemos decir que ya a finales del siglo XIX Gayet, en Francia, observó lesiones del mesencéfalo en una autopsia de un caso de letargia crónica. Más conocidos son los hallazgos de Von Economo ${ }^{13}$, que describe en la I Guerra Mundial, lesiones del hipotálamo posterior y del mesencéfalo rostral en casos de encefalitis letárgica. Son clásicos los trabajos de Bremer ${ }^{14}$ en los años treinta del siglo XX, en que secciona el sistema nervioso central del gato, entre bulbo y médula ("encephale isolé"), y observa alternancias del sueño y la vigilia. Pero si la sección era más craneal ("cerveau isolé"), el animal entraba en un estado de sueño total. En base a los experimentos, Bremer afirmaba que lo que provocaría el sueño sería una desaferentización del sistema nervioso central, una interrupción de las vías ascendentes.

A principios del siglo XX, Claparède ${ }^{15}$ sugirió que el sueño era provocado por una intoxicación del organismo. Partidarios de esta teoría fueron Hess y Moruzzi, así como Piéron, anteriormente a Claparède. Pavlov ${ }^{16}$, en 1923 aplica su teoría del reflejo condicionado a la explicación de los fenómenos del sueño.

Realmente es von Ecónomo ${ }^{17}$ quien defiende la existencia de centros neurales responsables de la mecánica del sueño: habla de un centro situado en hipotálamo posterior y porción rostral del mesencéfalo, cuya lesión produce hipersomnolencia y estupor; y de un centro situado en hipotálamo anterior, para los estados de sueño. Hess $^{18}$ confirmó las hipótesis de von Ecónomo, pero con una salvedad, realmente era el tálamo el que contenía el verdadero centro del sueño, ya que la estimulación de su zona anteromedial (a baja latencia) producía estado de sueño.

La invención del electroencefalograma por parte de Berger ${ }^{19}$, inicia una nueva era en el estudio de los mecanismos de la vigilia y el sueño. Gracias a estos progresos pueden explicarse los trabajos de Bremer, y en este sentido, también son clásicos los trabajos de Magoun y Rhines ${ }^{20}$ y de Moruzzi y Magoun ${ }^{21}$, que llevan a concluir que la formación reticular caudal tendría que ver con el estado de sueño, y la craneal, con el de vigilia, correspondiéndose asimismo con datos obtenidos por Rhines y Magoun $^{22}$, referidos a la influencia de la formación reticular sobre las neuronas motoras de la médula. En la década de los cincuenta, Aserinsky y Kleitman $^{5}$ y su grupo $^{23,24}$ describen la etapa del sueño REM, en la que hay una inhibición del tono muscular, según demostrara por esos años Jouvet, así como una "paradójica" desincronización electroencefalográfica. Jouvet $^{25}$ pensaba que el centro responsable del mecanismo ejecutor del sueño REM era la porción rostral de la formación reticular pontina oral. Jouvet fue el que bautizó al sueño REM como sueño paradójico. Sin embargo, Reinoso ${ }^{26}$ ha demostrado con gran precisión que el centro que regula, como "director de orquesta", la fase de sueño REM, se halla en la región ventral paramediana de la formación reticular pontina oral, zona por otra parte importante en la regulación de movimientos oculares complejos.

Es en los años 80 del pasado siglo cuando se empieza sospechar de la importancia del tálamo en los fenómenos del sueño. En este sentido, son cruciales los trabajos de Villablanca y $\operatorname{col}^{27}$ que observan cómo el gato diencefálico (en que la corteza cerebral era ablacionada) presentaba la misma patología que el gato atalámico. Por ello, Villablanca pensó en la importancia de los circuitos corticotalámicos en la regulación del sueño. Por esa época, Steriade $^{28}$ demuestra la importancia del núcleo reticular del tálamo en la generación de espigas en el sueño.

El insomnio familiar grave es una enfermedad de tipo priónico descrita por Lugaresi en los años ochenta del pasado siglo ${ }^{4,29,30}$. En esta entidad, se da una imposibilidad para iniciarse el período de sueño lento, junto con alteraciones en el sueño REM. En el inicio se afectan los núcleos dorsomedial y anteriores del tála- 
mo y en sus periodos finales, se afecta la corteza cerebral, y especialmente las cortezas cingular y prefrontal. En cambio, en la enfermedad de Creutzfeldt-Jakob, también de tipo priónico y descrita a principios del siglo XX, las lesiones comienzan en corteza, y termina por afectarse el tálamo. Gracias a Lugaresi y su equipo, podemos hablar de la importancia del núcleo dorsomedial del tálamo en la instauración del sueño profundo.

En lo que a nosotros respecta (Figs. 2, 3 y 4), hemos visto que los citados núcleos talámicos dorsomedial y anteriores tienen conexiones específicas, que pueden explicar en parte la fisiopatología del insomnio familiar grave $\mathrm{e}^{31-33}$.

Gracias a los estudios referidos y a otros, cuya larga lista sería prolijo relatar, hoy día se sabe que en la regulación del ciclo vigilia-sueño intervienen diversas estructuras del sistema nervioso central, actuando como una compleja red neural, cuyo fisiologismo íntimo está sin embargo todavía por aclarar. Decía Cajal ${ }^{34}$ : "Pasarán siglos y acaso millares de años antes que el hombre pueda entrever algo del insondable arcano del mecanismo no sólo de nues- tra psicología, sino de la más sencilla, de un insecto". Cajal tiene un trabajo relacionado con el tema que nos ocupa, titulado "Las teorías sobre el ensueño", de $1908^{35}$. En el trabajo, cita a Duval, que en 1895 dice que en el sueño, y en base a los hallazgos de Cajal, habría una retracción de las ramificaciones nerviosas, interrumpiendo así la corriente, de lo cual no es partidario Cajal, pues, según él, las arborizaciones terminales tienen siempre la misma extensión, forma y grado de aproximación a los cuerpos celulares, cualquiera que sea el modo de muerte del animal. En cambio es partidario de que las células neuróglicas perivasculares tendrían la misión de ampliar los espacios vasculares, para provocar alteraciones en la congestión vascular, en relación a los procesos mentales. "Estas contracciones (de la neuroglia) pueden producirse automáticamente, pero más a menudo son provocadas por el estímulo de la voluntad, que logra de esta suerte, y obrando sobre un grupo particular de células neuróglicas, llevar el proceso de la asociación en direcciones determinadas." También dice que no ignora que esta hipótesis puede ser susceptible de ser modifi$\operatorname{cada}^{36}$. Aunque no exactamente, sí se



Figura 2. Proyecciones aferentes de los núcleos dorsomedial (MD) y anteriores (A) del tálamo del gato. BP: prosencéfalo basal; BS: tronco del encéfalo; Cx: corteza cerebral; Hpt: hipotálamo; MB: cuerpo mamilar; RT: núcleo reticular del tálamo. 


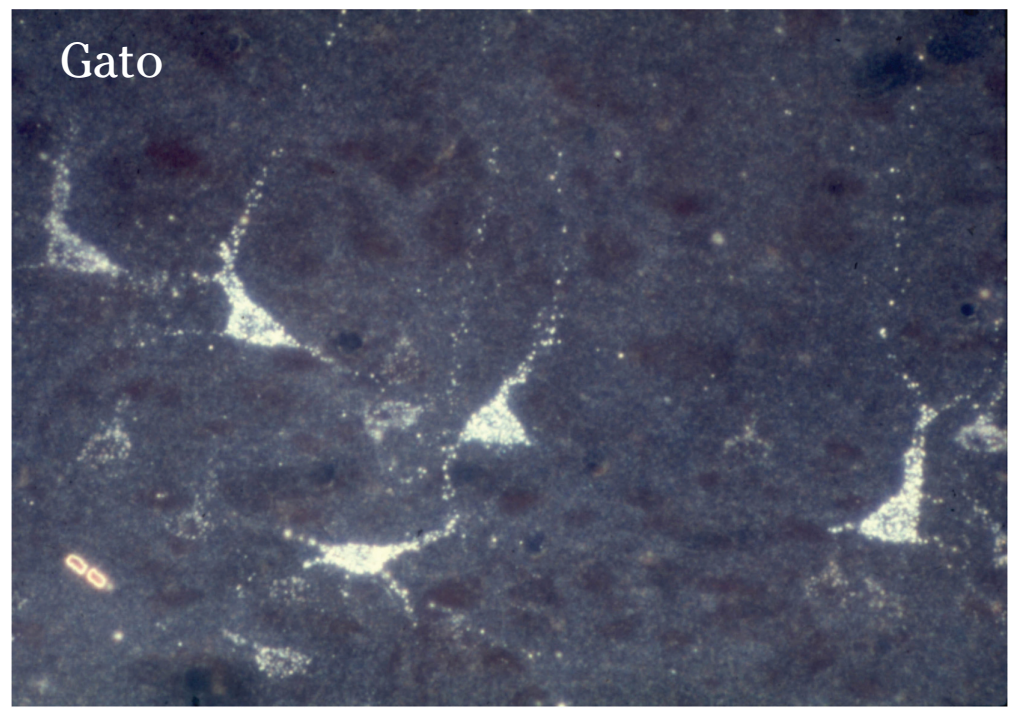

Figura 3. Cuerpos neuronales marcados retrógradamente, situados en las capas profunda de la corteza prefrontal, tras la inyección de horseradishperoxidase en el núcleo dorsomedial del tálamo del gato.

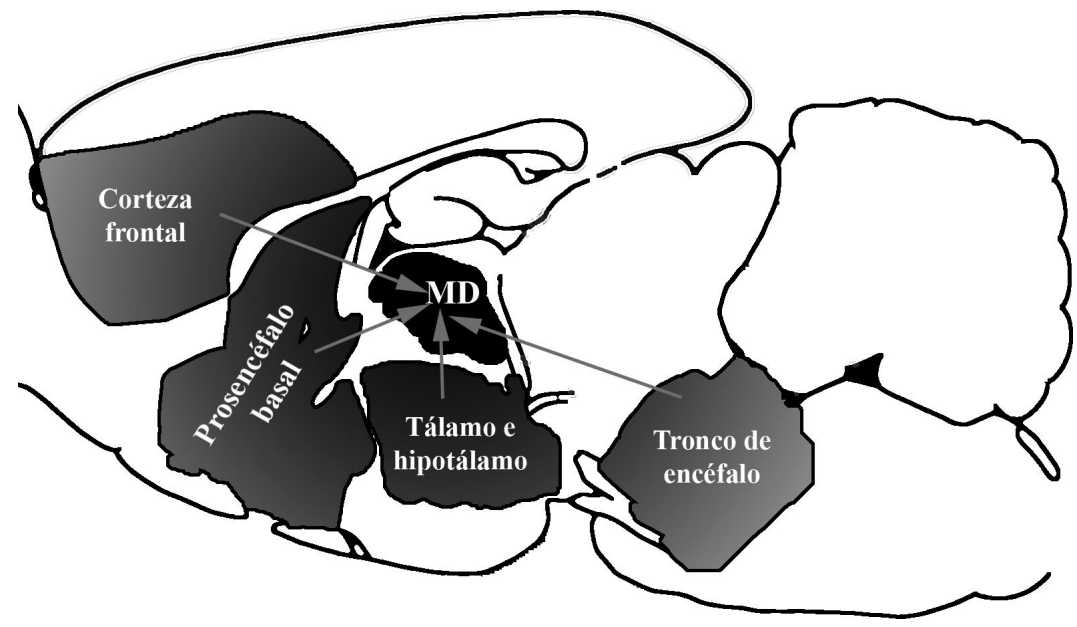

Figura 4. Proyecciones aferentes del núcleo dorsomedial (MD) de la rata. Estudio realizado tras la inyección de Fluorogold.

puede decir que hoy día se da una importancia más relevante que antes a los astrocitos, por su participación en la modulación de la transmisión sináptica ${ }^{37}$. Analizando concretamente los fenómenos oníricos, dice Cajal que "el ensueño perte- nece en la inmensa mayoría de los casos a la esfera visual". "Los ensueños auditivos y tactiles son menos frecuentes, y excepcionales los olfativos" ${ }^{\prime 35}$. Se presentan preferentemente durante la madrugada, y se trata de imágenes no sujetas a mecanis- 
mos asociativos del estado vigil. "El alborear de la razón y del sentido crítico marcan de ordinario el ocaso del sueño y anuncia el próximo despertar"35. También afirma que los ensueños son ajenos a las preocupaciones del día o a una actividad intelectual enérgica. Por introspección paciente, autosugestionándose, con el fin de analizar algunas facetas del sueño, observó que hay ensueños con relieve, con sensación de ejercer la convergencia ocular; que el color puede alcanzar la misma vivacidad que la realidad exterior; también afirma que el campo de la imagen del ensueño no varía, aunque se muevan los ojos o la cabeza. (Hoy se sabe bien que la participación de las redes neurales que controlan el mecanismo es esencial). Y cita Cajal observaciones de otros autores y de él mismo, que relatan cómo los ciegos tardíos sueñan con imágenes ópticas, aun careciendo de retina y nervio óptico, lo que confirma una vez más que se engendran en el propio sistema nervioso central.

Se puede decir, de acuerdo a los hallazgos realizados hasta ahora, que de la vigilia son responsables los grupos serotonérgicos del tronco del encéfalo (núcleos del rafe y otras neuronas serotonérgicas dispersas) ${ }^{38}$, grupos aminérgicos tales como el locus coeruleus, la sustancia negra y el área tegmental ventral de Tsai, neuronas histaminérgicas y orexinérgicas del hipotálamo posterior (sustancias estas últimas, descubiertas en 1998$)^{39,40}$, el núcleo tegmentopedunculopontino, neuronas glutamatérgicas del tegmento pontomesencefálico y del hipotálamo lateral y posterior, neuronas gabaérgicas (como moduladoras de todo el sistema $)^{41}$. Todas ellas, neuronas cuya actuación se realiza de forma importante sobre la corteza cerebral, en sus diferentes capas, y de un modo específico para cada neurotransmisor.

En cuanto al sueño NREM, y en lo que respecta a la fase de somnolencia, ya hemos dicho que es importante el núcleo reticular del tálamo, para la instauración de los husos de sueño. Según demostraran Sterman y Clemente ${ }^{42}$, el prosencéfalo basal es una estructura hipnogénica importante en el establecimiento del sueño profundo, pero también lo es el tálamo, y en concreto el núcleo dorsomedial, como demostraron Lugaresi y su grupo; también lo son las conexiones corticotalámicas, según demostrara Villablanca ${ }^{27}$. El núcleo reticular del tálamo, como pacemaker diencefálico, es esencial para el establecimiento de los husos de sueño ${ }^{28,43}$.También es importante el tronco del encéfalo ${ }^{44}$.

En cuanto al sueño REM, ya hemos dicho que es el núcleo reticular pontino oral en su zona ventral y paramediana el "director de orquesta" que regula la situación ${ }^{26}$, estando implicadas otras estructuras, interconectadas en una compleja red. Parece ser que se atribuyó a la acetilcolina la responsabilidad de la desincronización del EEG que se da en el sueño REM, ya que la inyección de carbacol en el tronco del encéfalo desencadena el sueño REM. Sin embargo, el grupo de Reinoso observó que cuando se inyectaba el carbacol en la porción ventral paramediana del núcleo reticular pontino oral, se producía el REM, pero cuando se inyectaba en el tegmento dorsal, se producía atonía, que es una de las características del REM ${ }^{45}$. También el citado grupo ha observado cómo la supresión de las neuronas serotonérgicas del núcleo dorsal del rafe y la activación de las neuronas colinérgicas del tegmento mesopontino, da lugar no sólo a la inhibición de todas estas estructuras, sino de otras estructuras del tallo cerebral y del prosencéfalo, que a su vez pueden activar o inhibir la mencionada zona del núcleo reticular pontino oral.

Así pues, dentro de la compleja red neural que regula los mecanismos del ciclo vigilia-sueño, son importantes la corteza cerebral, el prosencéfalo basal, el tálamo, el tronco del encéfalo, entre otras. Se pueden distinguir dos procesos, en cierta medida independientes dentro del sueño: la fase NREM, con sus implicaciones hodológicas propias, y la del REM, con otras implicaciones diversas; estando NREM y REM interrelacionados de forma rítmica, independientemente del ritmo sueño-vigilia-sueño en sí. El proceso de la vigilia presenta sus mecanismos propios, relacionados con el proceso del sueño, en forma homeostática y cronológicamente establecida.

Hoy día se da una gran importancia a las cortezas asociativas en el hombre, 
muy desarrolladas. Precisamente, una lesión de la corteza occipitotemporal da lugar a anoniria ${ }^{46}$.

\section{BIBLIOGRAFÍA}

1. McCarley RW. Sleep, dreams and states of consciousness. En: P. M. Conn. Neuroscience in medicine. Philadelphia, 1995: 535554.

2. Steriade M, McCormick, DA,SEJnowski TJ. Thalamocortical oscillations in the sleeping and aroused brain. Science 1993; 262: 679685.

3. Purves D, Augustine GJ, Fitzpatrick, D, Katz, LC, LAMANTIA, AS AND McNAMARA, J. Invitación a la neurociencia. Editorial Médica Panamericana. Buenos Aires, 2001.

4. Lugaresi E, MEdori R, Montagna P, Baruzzi A, Cortelli P, Lugaresi A, et al. Fatal familial insomnia and dysautonomia with selective degeneration of thalamic nuclei. $\mathrm{N}$ Engl $\mathrm{J}$ Med 1986; 315: 997-1003.

5. AsERINSKY E, KLEITMAN N. Regularly occurring periods of eye motility, and concomitant phenomena, during sleep. Science 1953; 118 : 273-274.

6. MOORE RY. Neural control of the pineal gland. Behav Brain Res 1996; 73: 125-130.

7. Teclemariam-Mesbah R, Ter Horst GJ, Postema F, WORTel J, Buiss RM. Anatomical demonstration of the suprachiasmatic nucleuspineal pathway. J Comp Neurol 1999; 406: 171-182.

8. Kalsbeek A, Teclemariam-Mesbah R, Pevet P. Efferent projections of the suprachiasmatic nucleus in the golden hamster (Mesocricetus auratus). J Comp Neurol 1993; 332: 293314.

9. Hobson JA, McCarley RW. The brain as a dream state generator: an activation-synthesis hypothesis of the dream process. Am J Psychiatry 1977; 134: 1335-1348.

10. Hobson JA. The chemistry of conscious state. Editor. Boston, 1994.

11. Hobson JA, Stickgold R. The conscious state paradigm: a neurocognitive approach to waking, sleeping and dreaming. En: M. Gazzaniga. The cognitive neurosciences. Cambridge, Mass., 1995: 1373-1389.

12. ReINoso-SuAREZ F. Investidura como Doctor Honoris Causa. EUNSA. Madrid, 2002.

13. Von Economo C, En Brodal, A. The reticular formation and some related nuclei. En: Oxford University Press. Neurological anato- my in relation to clinical medicine. New York, 1981.

14. BREMER F. Cerveau "isolé" et physiologie du sommeil. C.R. Soc. Biol. 1935; 118: 1235-1241.

15. Claparede E. Esquisse d'une théorie biologique du sommeil. Arch Psychol 1905; 4: 246-349.

16. PAvlov IP. "Innere Hemmung" der bedingten. Reflexe und der Schlaf - ein und derselbe Proze. Skand Arch Physiol 1923; 44: 42-58.

17. Von Economo C. Encephalitis lethargica. Wien Med Wischr 1923; 73: 777-782, 835-838, 1113-1117, 1243-1249, 1334-1338.

18. HEss WR. Das Schlafsyndrom als Folge dencephaler Reizung. Helv Physiol Pharmacol Acta 1944; 2: 305-344.

19. BERGER H. Über das Elektroenkephalogramm des Menschen. J Psychol Neurol 1930; 40: 160-179.

20. Magoun HW, Rhines R. An inhibitory mechanism in the bulbar reticular formation. Journal of Neurophysiology 1946; 9: 165-171.

21. Moruzzi G, Magoun HW. Brain stem reticular formation and activation of the EEG. 1949. J Neuropsychiatry Clin Neurosci 1995; 7: 251267.

22. Rhines R, MAGOun HW. Brainstem facilitation of cortical motor responses. Journal of Neurophysiology 1946; 9: 219-229.

23. Dement W, Kleitman N. Cyclic variations in EEG during sleep and their relation to eye movements, body motility, and dreaming. Electroencephalogr Clin Neurophysiol Suppl 1957; 9: 673-690.

24. DEMENT W, WolPERT EA. The relation of eye movements, body motility, and external stimuli to dream content. J Exp Psychol 1958; 55: 543-553.

25. JOUvET M. Recherches sur les structures nerveuses et les mécanismes responsales des différentes phases du sommeil physiologique. Arch Ital Biol 1962; 100: 125-206.

26. De La Roza C, Reinoso-Suarez F. Ultrastructural synaptic organization of axon terminals in the ventral part of the cat oral pontine reticular nucleus. J Comp Neurol 2000; 427: 31-53.

27. Villablanca JR, De AndRes I, Olmstead CE. Sleep-waking states develop independently in the isolated forebrain and brain stem following early postnatal midbrain transection in cats. Neuroscience 2001; 106: 717-731.

28. Steriade M, Dossi RC, Nunez A. Network modulation of a slow intrinsic oscillation of cat thalamocortical neurons implicated in sleep delta waves: cortically induced synchroniza- 
tion and brainstem cholinergic suppression. J Neurosci 1991; 11: 3200-3217.

29. Lugaresi E, Montagna P. Thalamus, sleep and circadian functions. En: M. Mancia and G. Marini. The diencephalon and sleep. New York, 1994: 215-220.

30. LUGARESI E, OMICINI L. El sueño, los sueños, un mundo misterioso. EUNSA. Pamplona, 1999.

31. Velayos JL, Reinoso-Suarez F. Prosencephalic afferents to the mediodorsal thalamic nucleus. J Comp Neurol 1985; 242: 161-181.

32. Velayos JL, Oliva M, Alfageme F. Afferent projections to the mediodorsal and anterior thalamic nuclei in the cat. Anatomical-clinical correlations. Brain Pathol 1998; 8: 549552.

33. Velayos Jl, Ullán J, Amat A, Ramos P, Sieira JA. Expression of the cellular prion protein $\left(\mathrm{PrP}^{\mathrm{C}}\right)$ in the cat central nervous system. Some findings. Eur J Anat 2002; 6: 23-29.

34. RAMÓn y CAJAL S. Recuerdos de mi vida: historia de mi labor científica. Alianza Editorial. Madrid, 1981.

35. RAMÓN Y CAJAL S. Las teorías sobre el ensueño. Revista de Medicina y Cirugía de la Facultad de Medicina de Madrid. 1908; II: 8798.

36. RAMÓN Y CAJAL S. Sobre las relaciones de las células nerviosas con las neuróglicas. Revista trimestral micrográfica 1896; 1:123126.

37. Perea G, Araque A. Nuevas vías de información en el sistema nervioso: comunicación entre astrocitos y neuronas. Rev Neurol 2003; 36: 137-144.
38. Rodrigo-Angulo ML， Rodriguez-Veiga E, Reinoso-SuAREZ F. Serotonergic connections to the ventral oral pontine reticular nucleus: implication in paradoxical sleep modulation. J Comp Neurol 2000; 418: 93-105.

39. Sakurai T, AmemiYa A, Ishi M, MatsuZaKi I, Chemelli RM, TANaKa $\mathrm{H}$ et al. Orexins and orexin receptors: a family of hypothalamic neuropeptides and $\mathrm{G}$ protein-coupled receptors that regulate feeding behavior. Cell 1998; 92: 573-585.

40. Sutcliffe JG, DE Lecea L. The hypocretins: setting the arousal threshold. Nat Neurosci 2002; 3: 339-349.

41. KING DP, TALAHASHI JS. Molecular mechanisms of circadian rhythms in mammals. Annu Rev B Neurosci 2000; 23: 713-742.

42. Sterman MB, Clemente CD. Forebrain inhibitory mechanisms: sleep patterns induced by basal forebrain stimulation in the behaving cat. Exp Neurol 1962; 6: 103117.

43. Sillito AM, Jones HE, Gerstein GL, West DC. Feature-linked synchronization of thalamic relay cell firing induced by feedback from the visual cortex. Nature 1994; 369: 479-482.

44. FoRCADAS MI, ZARRANZ JJ. Insomnio y alucinaciones tras lesiones vasculares del tegmento protuberancial en el hombre. Neurología 1994; 9: 211-223.

45. REINOSO-SuÁrez F, DE ANDRÉs I, RODRIGO-ANGULO M, GARZón M. Brain structures and mechanisms involved in the generation of REM slepp. Sleep Med Rev 2001; 5: 63-78.

46. Poza JJ, Marti Masso JF. Pérdida completa de ensoñaciones tras una lesión cerebral temporooccipital. Neurologia 2006; 21:152-154. 
